\title{
SEMIGROUPS OF HIGH RANK
}

\author{
by EMILIA GIRALDES and JOHN M. HOWIE
}

(Received 1st November, 1983)

\section{Introduction}

By the $\operatorname{rank} r(S)$ of a finite semigroup $S$ we shall mean the minimum cardinality of a set of generators of $S$. For a group $G$, as remarked in [3], one has $r(G) \leqq \log _{2}|G|$, the bound being attained when $G$ is an elementary abelian 2-group. By contrast, we shall see that there exist finite semigroups $S$ for which $r(S) \geqq|S|-1$. In the hope that it will not be considered too whimsical, we shall refer to a finite semigroup $S$ of maximal rank (i.e. for which $r(S)=|S|$ ) as royal; a semigroup of next-to-maximal rank (i.e. for which $r(S)=|S|-1)$ will be called noble.

It is possible to extend these ideas to infinite semigroups by defining $S$ to be royal if $\langle S \backslash\{s\}\rangle \subset S$ (properly) for all $s$ in $S$. Equally, $S$ is said to be noble if $\left\langle S \backslash\left\{s_{1}, s_{2}\right\}\right\rangle \subset S$ for all $s_{1} \neq s_{2}$ in $S$, but there exists $z$ in $S$ such that $\langle S \backslash\{z\}\rangle=S$. The element $z$ featuring in this definition will be called a superfluous element.

The structure of royal semigroups, which are necessarily bands, is given by Theorem 2.2. A noble semigroup $S$ is defined as singly or doubly noble according as $S$ has exactly one or exactly two superfluous elements. (As is shown in Theorem 3.7, there are no other possibilities.) The main part of this paper is devoted to an elucidation of the structure of singly noble semigroups. These need not be bands, but the description in Section 4 of singly noble bands turns out to be a major step in understanding the structure of singly noble semigroups in general. The main results are Theorems 4.11, $4.14,4.15,5.6$ and 5.10 .

Unexplained terms in semigroup theory will be found in [2].

\section{Royal semigroups}

Let $S$ be a royal semigroup. If $a \neq a^{2}$ in $S$ then $S \backslash\left\{a^{2}\right\}$ generates $S$, contrary to assumption. Hence $S$ is a band and so by the Clifford-McLean Theorem $[1,4,2]$ is a semilattice $Y$ of rectangular bands $E_{\alpha}(\alpha \in Y)$ :

$$
S=\mathscr{P}\left[Y ;\left\{E_{\alpha}: \alpha \in Y\right\}\right] .
$$

We now have a lemma which applies also to noble bands.

Lemma 2.1. Let $S=\mathscr{S}\left[Y ;\left\{E_{\alpha}: \alpha \in Y\right\}\right]$ be a royal or noble band. Then each $E_{\alpha}$ is either a left zero or a right zero semigroup. 
Proof. Denoting the class of right zero semigroups by $\mathbf{R Z}$ and the class of left zero semigroups by $\mathbf{L Z}$, let us suppose by way of contradiction that some $E_{\alpha}$ does not belong to $\mathbf{R Z} \cup \mathbf{L Z}$. Then $E_{\alpha}$ contains a copy $\left\{e_{11}, e_{12}, e_{21}, e_{22}\right\}$ of the $2 \times 2$ rectangular band, in which

$$
e_{i j} e_{k l}=e_{i l} .
$$

Since $e_{12}=e_{11} e_{22}$ and $e_{21}=e_{22} e_{11}$ it is clear that $S \backslash\left\{e_{12}, e_{21}\right\}$ generates $S$, contrary to hypothesis.

The next observation is that if $S=\mathscr{S}\left[Y ;\left\{E_{\alpha}: \alpha \in Y\right\}\right]$ is royal then $Y$ is a chain. For if $Y$ has a branch-point $\gamma$, i.e. a point for which there exist $\alpha, \beta>\gamma$ such that $\alpha \beta=\gamma$, then $E_{\alpha} E_{\beta} \subseteq E_{\gamma}$ and so there exists at least one element $z$ of $E_{y}$ having an expression $z=x y$ with $x$ in $E_{\alpha}, y$ in $E_{\beta}$ and so certainly with $x \neq z, y \neq z$. Thus $S \backslash\{z\}$ generates $S$, contrary to assumption.

In stating the first main theorem it is convenient to use the symbol $\Omega$ to denote the class of all non-zero cardinal numbers. We shall also use the symbols $R, L$ to stand for right and left, in a way that will be clear.

Theorem 2.2. Let $(Y, \leqq)$ be a chain and let $M: Y \rightarrow \Omega, H: Y \rightarrow\{R, L\}$ be maps. For each $\alpha$ in $Y$ let $E_{\alpha}$ be a set with cardinality $M(\alpha)$, endowed with the structure of a right zero or a left zero semigroup according as $H(\alpha)=R$ or $H(\alpha)=L$. Define a multiplication on $S=\bigcup\left\{E_{\alpha}: \alpha \in Y\right\}$ by the rule that

$$
x y=y x=y\left(x \in E_{\alpha}, y \in E_{\beta}, \alpha>\beta\right) .
$$

Then $S=\operatorname{Roy}(Y, M, H)$ is a royal semigroup.

Conversely, every royal semigroup is isomorphic to one constructed in this way.

Proof. For the direct part, it is a routine matter to show that the multiplication on $S=\bigcup\left\{E_{\alpha}: \alpha \in Y\right\}$ is associative and that $S$ is royal. As regards the converse, we have already seen that a royal semigroup $S$ must be a chain $Y$ of semigroups $E_{\alpha}$ in $\mathbf{R Z} \cup \mathbf{L Z}$. We may therefore define (for each $\alpha$ in $Y$ )

$$
M(\alpha)=\left|E_{\alpha}\right|, \quad H(\alpha)=\left\{\begin{array}{lll}
R & \text { if } & E_{\alpha} \in \mathbf{R Z} \\
L & \text { if } & E_{\alpha} \in \mathbf{L Z},\left|E_{\alpha}\right|>1 .
\end{array}\right.
$$

For any $x, y$ in $S$ we must have either $x y=x$ or $x y=y$, since otherwise $S \backslash\{x y\}$ would generate $S$. So if we now take $\alpha>\beta, x \in E_{\alpha}, y \in E_{\beta}$ we know that

$$
E_{\alpha} E_{\beta} \subseteq E_{\beta}, \quad E_{\beta} E_{\alpha} \subseteq E_{\beta}
$$

and so the only possibility is

$$
x y=y x=y .
$$


Remark. It is easy to verify that every subsemigroup and every homomorphic image of a royal semigroup is royal. The class Roy of royal semigroups is not, however, a variety, since a direct product of royal semigroups need not be royal. The class Roy is contained in the variety $\left[x^{2}=x, a x y a=a x a y a\right]$ of regular bands. See Petrich [5] for information on varieties of bands.

\section{Noble semigroups: preliminaries}

Let $S$ be a noble semigroup, generated by $S \backslash\{z\}$. As in the introduction, we refer to $z$ as a superfluous element. It is clear that for all $s$ in $S \backslash\{z\}$

$$
s^{2}=s \quad \text { or } \quad s^{2}=z
$$

for any other value for $s^{2}$ would imply that $S \backslash\left\{s^{2}, z\right\}$ generates $S$. More generally, and for the same reason,

$$
s t=s \quad \text { or } \quad s t=t \quad \text { or } \quad s t=z
$$

for all $s, t$ in $S \backslash\{z\}$.

Also trivial is

Lemma 3.3. Let $S$ be a noble semigroup, generated by $S \backslash\{z\}$. Then there exist $g, h$ in. $S \backslash\{z\}$ such that $g h=z$.

Proof. Otherwise $S \backslash\{z\}$ is closed under multiplication and does not generate $S$.

The next result is not quite so obvious:

Lemma 3.4. Let $S$ be a noble semigroup, generated by $S \backslash\{z\}$. Then $z^{2}=z$.

Proof. Suppose by way of contradiction that $z^{2}=g(\in S \backslash\{z\})$. Then

$$
g z=z g\left(=z^{3}\right)
$$

If $S=\{g, z\}$ is of order 2 then $g^{2}=z$ by Lemma 3.3 and either $g z=z g=g$ or $g z=z g=z$. In the former case

$$
g^{2} z=z^{2}=g, \quad g(g z)=g^{2}=z
$$

which contradicts associativity; in the latter case associativity again fails, since

$$
z^{2} g=g^{2}=z, \quad z(z g)=z^{2}=g .
$$

Accordingly, suppose that $|S| \geqq 3$, and let $h \in S \backslash\{g, z\}$. By (3.1) we have either $h^{2}=z$ or $h^{2}=h$. In fact we must have $h^{2}=h$, for $h^{2}=z$ implies that $h^{4}=g$ and hence that $S \backslash\{g, z\}$ generates $S$. Thus all elements of $S \backslash\{g, z\}$ are idempotent. 
Continuing with our supposition that $z^{2}=g$ we now consider two cases: (1) $g^{2}=g$; (2) $g^{2}=z$. In case (1) we consider the expression $g_{1} g_{2}$ for $z$. Such an expression must exist (with $g_{1}, g_{2} \in S \backslash\{z\}$ ) by Lemma 3.3. If both $g_{1}, g_{2}$ are distinct from $g$ then

$$
g=z^{2}=\left(g_{1} g_{2}\right)^{2}
$$

implies that $S \backslash\{z, g\}$ generates $S$. Hence we have either

$$
z=g g_{2} \quad \text { or } \quad z=g_{1} g
$$

In the former case we get

$$
g z=g\left(g g_{2}\right)=g^{2} g_{2}=g g_{2}=z
$$

and hence

$$
z^{2}=z g g_{2}=g z g_{2}=z g_{2}=\left(g g_{2}\right) g_{2}=g g_{2}^{2}=g g_{2}=z,
$$

a contradiction to the assumption that $z^{2}=g$. A similar contradiction arises if we assume at (3.6) that $z=g_{1} g$. Thus case (1) cannot arise.

In case (2) it follows by (3.5) that

$$
(g z)^{2}=g^{2} z^{2}=z g=g z
$$

and so $g z$, being idempotent, must be distinct from both $g$ and $z$. This implies that $S \backslash\{z, g z\}$ generates $S$ and so again gives a contradiction. Thus case (2) cannot arise either. We have now shown as required that $z^{2}=z$.

Next, we have

Theorem 3.7. Let $S$ be a noble semigroup. Then either there is a unique superfluous element or there are precisely two such elements. In the latter case $S$ must be a band.

Proof. Suppose that $S \backslash\{z\}$ generates $S$. We"consider two cases:

(1) there exists $g$ in $S \backslash\{z\}$ such that $g^{2}=z$;

(2) $g^{2}=g$ for all $g$ in $S \backslash\{z\}$.

In case (1) the superfluous element $z$ must be unique. For suppose that $t(\neq z)$ is also superfluous; then $t^{2}=t$ by Lemma 3.4 and so $t \neq g$; hence $g, z \in S \backslash\{t\}$ with $g^{2}=z$ and so $g$ and $t$ violate condition (3.1).

In case (2) we note that by Lemma 3.4 the semigroup $S$ is a band. We now show that $S$ cannot have more than two superfluous elements. By Lemma 3.3 we have

$$
z=g h
$$

where $g, h \in S \backslash\{z\}$. In fact $g$ and $h$ must be distinct since $S$ is a band. It follows that

$$
g z=z=z h .
$$


The only possible candidates for alternative generating sets are $S \backslash\{g\}$ and $S \backslash\{h\}$; for if $s \notin\{z, g, h\}$ then $S \backslash\{s\}$ must contain all three of $z, g, h$ and so by (3.8) must violate (3.2). Let us now suppose that $S \backslash\{g\}, S \backslash\{h\}$ are both generating sets; we shall see that this leads to a contradiction. By Lemma 3.3 we have

$$
g=x y,
$$

where $x, y \in S \backslash\{g\}$. If $x, y$ are both in $S \backslash\{z\}$ we can immediately deduce that $S \backslash\{z, g\}$ generates $S$, in contradiction to our supposition that $S$ is noble. Hence either

$$
g=z y \quad \text { or } \quad g=x z,
$$

where $x, y \in S \backslash\{z, g\}$. Suppose first that $g=z y$. Then if $y \neq h$ the equation $g=z y$ (in which $g, z, y \in S \backslash\{h\}$ ) violates condition (3.2) for the generating set $S \backslash\{h\}$. Hence $y=h$. By the same token, if we assume that $g=x z$ in (3.10) we find that $x=h$. Hence we can refine (3.10) to

$$
g=z h \text { or } g=h z \text {. }
$$

In fact $g=z h$ is impossible because of (3.9), and so $g=h z$. But then (3.9) gives

$$
z=g z=h z^{2}=h z=g,
$$

which is again a contradiction. The only conclusion is that $S \backslash\{g\}$ and $S \backslash\{h\}$ cannot simultaneously be generating sets. This completes the proof of Theorem 3.7.

It is convenient now to define a noble semigroup $S$ as singly noble if it has a unique superfluous element, and doubly noble if it has precisely two such elements. We shall not discuss doubly noble semigroups in this paper. Such semigroups do, however, exist, as the following example makes clear.

Example. (3.11) Let $S=\left\{a_{1}, a_{2}, z_{1}, z_{2}\right\} \subseteq \mathscr{T}(\{1,2,3,4\})$, where

$$
\begin{array}{lll}
a_{1}=\left(\begin{array}{llll}
1 & 2 & 3 & 4 \\
1 & 1 & 4 & 4
\end{array}\right), & a_{2}=\left(\begin{array}{llll}
1 & 2 & 3 & 4 \\
2 & 2 & 3 & 3
\end{array}\right), \\
z_{1}=\left(\begin{array}{llll}
1 & 2 & 3 & 4 \\
3 & 3 & 3 & 3
\end{array}\right), & z_{2}=\left(\begin{array}{llll}
1 & 2 & 3 & 4 \\
4 & 4 & 4 & 4
\end{array}\right)
\end{array}
$$

Then $S$ has Cayley table

\begin{tabular}{l|llll} 
& $a_{1}$ & $a_{2}$ & $z_{1}$ & $z_{2}$ \\
\hline$a_{1}$ & $a_{1}$ & $a_{2}$ & $z_{1}$ & $z_{2}$ \\
$a_{2}$ & $a_{1}$ & $a_{2}$ & $z_{1}$ & $z_{2}$ \\
$z_{1}$ & $z_{2}$ & $z_{1}$ & $z_{1}$ & $z_{2}$ \\
$z_{2}$ & $z_{2}$ & $z_{1}$ & $z_{1}$ & $z_{2}$
\end{tabular}


and is clearly doubly noble, generated by either $S \backslash\left\{z_{1}\right\}$ or $S \backslash\left\{z_{2}\right\}$ but not by any subset of cardinality 2 .

\section{Singly noble bands}

A singly noble semigroup need not be a band, but, as will be clear in Section 5, the major step in understanding the structure of singly noble semigroups in general is the study of singly noble bands. We begin with what at first seems to be a very particular example.

Let $A$ be a right zero semigroup and let $Z$ be a left zero semigroup, where $|A| \geqq 1$, $|Z| \geqq 2$. Let $P$ be a subset of $Z$ such that $|P| \geqq 2$ and let $z_{1}$ be a fixed element of $P$. Define a multiplication on the disjoint union $S=A \cup Z$ by the rules

$$
\begin{gathered}
z a=z \\
a z \in Z, a \in A) \\
a z=\left\{\begin{array}{ll}
z_{1} & (a \in A, z \in P) \\
z & (a \in A, z \in Z \backslash P)
\end{array}\right\}
\end{gathered}
$$

It is possible to check directly that this is an associative multiplication. It is easy also to see that $S$ is a singly noble band, with superfluous element $z_{1}$ and with two $\mathscr{F}$-classes, namely $A$ and $Z$. The semigroup is in fact determined by the sets $A, Z$ and $P$ and by the fact that $A$ and $Z$ are respectively in $\mathbf{R Z}$ and $\mathbf{L Z}$. To draw attention to these facts and also to the unique superfluous element $z_{1}$ we write

$$
S=S N B_{L}^{R}\left(A, Z ; P ; z_{1}\right) .
$$

The dual semigroup, in which the upper $\mathscr{J}$-class is left zero and the lower $\mathscr{J}$-class is right zero, is denoted by

$$
S N B_{R}^{L}\left(A, Z ; P ; z_{1}\right) \text {. }
$$

In a very similar way we construct a singly noble band in the case where $A$ and $Z$ are both in RZ, where $P$ is a subset of $Z$ such that $|P| \geqq 2$ and where $z_{1} \in P$. Here the specification is

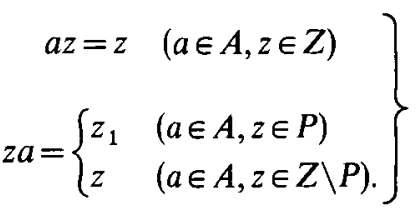

We write

$$
S=S N B_{R}^{R}\left(A, Z ; P ; z_{1}\right) .
$$

The dual semigroup, in which both $\mathscr{J}$-classes are in LZ, is written

$$
S N B_{L}^{L}\left(A, Z ; P ; z_{1}\right) \text {. }
$$


We can now prove the following theorem:

Theorem 4.7. Let $S$ be a singly noble band with superfluous element $z_{1}$ and with two $\mathscr{J}$-classes. Then (for some $\left.A, Z, P, z_{1}\right) S$ is isomorphic to exactly one of the semigroups listed in formulae (4.2), (4.3), (4.5) and (4.6).

Proof. By Lemma 2.1 the $\mathscr{f}$-classes of $S$ are both in $\mathbf{R Z} \cup \mathbf{L Z}$. They form a twoelement chain and it is clear that $z_{1}$ must be in the lower $\mathscr{J}$-class. Denote the $\mathscr{J}$-classes by $A, Z$, with

$$
A Z \subseteq Z, \quad Z A \subseteq Z
$$

If $|Z|=1$ the semigroup is simply a right or left zero semigroup $A$ with a zero element $z_{1}$ adjoined, and such a semigroup is royal rather than noble. Hence $|Z| \geqq 2$.

There are now four cases, according to whether $A$ and $Z$ are in $\mathbf{R Z}$ or in $\mathbf{L Z}$, but because of duality it will not be necessary to consider more than two. Suppose first that $A \in \mathbf{R Z}, Z \in \mathbf{L Z}$. Thus

$$
a a^{\prime}=a^{\prime}, \quad z z^{\prime}=z
$$

for all $a, a^{\prime}$ in $A$ and all $z, z^{\prime}$ in $Z$. The product $z_{1} a$ must be in $Z$ and cannot be $z \neq z_{1}$, since then $S \backslash\{z\}$ would generate $S$. Hence

$$
z_{1} a=z_{1}
$$

for all $z$ in $A$, and it now follows easily that for all $z$ in $Z$

$$
z a=\left(z z_{1}\right) a=z\left(z_{1} a\right)=z z_{1}=z .
$$

Now by the same argument as we used for $z_{1} a$ we must have

$$
a z_{1}=z_{1}
$$

for all $a$ in $A$. Since $S \backslash\left\{z_{1}\right\}$ generates $S$ there must exist $a_{0}$ in $A$ and $z_{0}\left(\neq z_{1}\right)$ in $Z$ such that

$$
a_{0} z_{0}=z_{1} .
$$

We now show that for each $z\left(\neq z_{1}\right)$ in $Z$ we have the implication

$$
A z \cap\left\{z_{1}\right\} \neq \emptyset \Rightarrow A z=\left\{z_{1}\right\} .
$$

For suppose that $a^{\prime} z=z_{1}$ for some $a^{\prime}$ in $A$. Then for all $a$ in $A$ we have $a z \in\left\{z, z_{1}\right\}$. If $a z=z$ then

$$
z=a z=\left(a^{\prime} a\right) z=a^{\prime}(a z)=a^{\prime} z=z_{1},
$$


which is a contradiction. Hence $a z=z_{1}$ for all $a$ in $A$. We have proved the implication (4.10).

It follows that $Z$ divides into two complementary subsets $P$ and $Z \backslash P$ given by

$$
\begin{gathered}
P=\left\{z \in Z: A z=\left\{z_{1}\right\}\right\}, \\
Z \backslash P=\left\{z \in Z: z \neq z_{1}, A z=\{z\}\right\} .
\end{gathered}
$$

By (4.8) and (4.9) $z_{1}$ and $z_{0}$ belong to $P$. Hence $|P| \geqq 2$.

We have in fact shown that

$$
S \simeq S N B_{L}^{R}\left(A, Z ; P ; z_{1}\right) .
$$

Suppose now that the $\mathscr{J}$-classes $A$ and $Z$ are both in $\mathbf{R Z}$, so that

$$
a a^{\prime}=a^{\prime}, \quad z z^{\prime}=z^{\prime}
$$

for all $a, a^{\prime}$ in $A$ and all $z, z^{\prime}$ in $Z$. Then as before we must have $a z_{1}=z_{1} a=z_{1}$ for all $a$ in $A$ and so

$$
a z=a\left(z_{1} z\right)=\left(a z_{1}\right) z=z_{1} z=z
$$

for all $a$ in $A$ and $z$ in $Z$. Again there exist $z_{0}\left(\neq z_{1}\right)$ in $Z$ and $a_{0}$ in $A$ such that

$$
z_{0} a_{0}=z_{1}
$$

and once again we can show that for each $z \neq z_{1}$ either $z A=\left\{z_{1}\right\}$ or $z A=\{z\}$. Thus again we find

$$
\begin{gathered}
P=\left\{z \in Z: z A=\left\{z_{1}\right\}\right\}, \\
Z \backslash P=\left\{z \in Z: z \neq z_{1}, z A=\{z\}\right\},
\end{gathered}
$$

with $z_{0}, z_{1} \in P$. Thus

$$
S \simeq S N B_{R}^{R}\left(A, Z ; P ; z_{1}\right)
$$

Note. In (4.2), (4.3), (4.5) and (4.6) we are insisting that $P$ properly contains $\left\{z_{1}\right\}$. If we allow $P=\left\{z_{1}\right\}$ then the semigroup in each case becomes royal. We shall sometimes want to allow this degenerate case.

We have effectively described all singly noble bands with two $\mathscr{J}$-classes. Let us now consider an arbitrary singly noble band

$$
S=\mathscr{S}\left[Y ;\left\{E_{\alpha}: \alpha \in Y\right\}\right],
$$


generated by $S \backslash\{z\}$. As pointed out in the paragraph preceding the statement of Theorem 2.2, at any branch-point $\gamma$ of the semilattice $Y$ there must be at least one element in $E_{j}$ expressible as a product of two elements distinct from itself. In the case of a royal semigroup the effect of this observation was to conclude that $Y$ must be a chain. Here we conclude that $Y$ can have at most one branch-point, and that the branch-point (if any) must be at the $\mathscr{J}$-class containing $z$. Whether or not a branch-point occurs it will be useful to refer to the $\mathscr{J}$-class containing $z$ as the pivotal $\mathscr{J}$-class. The element $z$ itself will be called the pivot. If $Y$ is a chain we shall say that $S$ is a catenary singly noble band.

Let us first consider the catenary case and denote the pivotal $\mathscr{J}$-class by $E_{0}$; then 0 is not the maximum element of the chain $Y$. The structure of $E_{\alpha} \cup E_{\beta}(\alpha>\beta)$ must be that of a royal semigroup if $\beta \neq 0$. The structure of $E_{\alpha} \cup E_{0}(\alpha>0)$ is either that of a royal semigroup, or for some $P \subseteq E_{0}$ with $|P| \geqq 2$ is the appropriate $\operatorname{SNB}\left(E_{\alpha}, E_{0} ; P ; z_{1}\right)$. We thus have a good deal of information about the global structure of $S$.

In fact we can be still more specific. Suppose that $\alpha>\beta>0$ and suppose for definiteness that

$$
E_{\alpha}, E_{\beta} \in \mathbf{R Z}, \quad E_{0} \in \mathbf{L Z}
$$

Suppose now that

$$
\begin{aligned}
& E_{\alpha} \cup E_{0}=S N B_{L}^{R}\left(E_{\alpha}, E_{0} ; P ; z_{1}\right), \\
& E_{\beta} \cup E_{0}=S N B_{L}^{R}\left(E_{\beta}, E_{0} ; Q ; z_{1}\right),
\end{aligned}
$$

with $|P|,|Q| \geqq 2$. Since

$$
E_{\alpha} \cup E_{\beta}=\operatorname{Roy}(\{\alpha, \beta\}, M, H)
$$

(with $M(\alpha)=\left|E_{\alpha}\right|, M(\beta)=\left|E_{\beta}\right|, H(\alpha)=H(\beta)=R$ ) we have that

$$
a b=b a=b
$$

for all $a$ in $E_{\alpha}, b$ in $E_{\beta}$. Hence, by (4.1), for $b$ in $E_{\beta}$ and $z$ in $P$,

$$
b z=(b a) z=b(a z)=b z_{1}=z_{1} .
$$

It follows that $z \in Q$, and so $P \subseteq Q$. The same result applies to other mixtures of right and left zero semigroups.

We can now state the following theorem. As in Theorem 2.2, $\Omega$ denotes the class of all non-zero cardinal numbers.

Theorem 4.11. Let $(Y, \leqq)$ be a chain and let 0 be a fixed non-maximal element of $Y$. Let $M: Y \rightarrow \Omega$ and $H: Y \rightarrow\{R, L\}$ be maps and suppose that $M(0) \geqq 2$. For each $\alpha$ in $Y$ suppose that $E_{\alpha}$ is a set with $M(\alpha)$ elements having right or left zero semigroup structure according as $H(\alpha)$ is $R$ or $L$. Let $z_{1}$ be a fixed element of $E_{0}$. 
For each $\alpha>0$ let $P(\alpha)$ be a subset of $E_{0}$, and suppose that

(i) $z_{1} \in P(\alpha)$ for all $\alpha>0$,

(ii) $0<\alpha \leqq \beta \Rightarrow P(\alpha) \supseteq P(\beta)$,

(iii) $|P(\alpha)| \geqq 2$ for some $\alpha>0$.

Define a multiplication on $E_{\alpha} \cup E_{\beta}(\alpha>\beta \neq 0)$ by the rule that

$$
E_{\alpha} \cup E_{\beta}=\operatorname{Roy}(\{\alpha, \beta\}, M|\{\alpha, \beta\}, H|\{\alpha, \beta\})
$$

and on $E_{\alpha} \cup E_{0}(\alpha>0)$ by the rule that

$$
E_{\alpha} \cup E_{0}=S N B_{H(0)}^{H(\alpha)}\left(E_{\alpha}, E_{0} ; P(\alpha) ; z_{1}\right)
$$

Then the disjoint union $S=\bigcup\left\{E_{\alpha}: \alpha \in Y\right\}$ is a catenary singly noble band.

Conversely, every catenary singly noble band is isomorphic to one constructed in this way.

Proof. In effect we have already established the more difficult converse half of this result, provided we make as in the proof of Theorem 2.2 the convention that $H(\alpha)=R$ (say) whenever $\left|E_{\alpha}\right|=1$. The condition $P \subseteq Q$ obtained just before the statement of the theorem translates into property (ii) of the function $P$. If $|P(\alpha)|=1$ for any $\alpha$ (giving $|P(\beta)|=1$ for all $\beta \geqq \alpha)$ then the corresponding

$$
S N B_{H(0)}^{H(\alpha)}\left(E_{\alpha}, E_{0} ; P(\alpha) ; z_{1}\right)
$$

reduces simply to $\operatorname{Roy}(\{\alpha, 0\}, M|\{\alpha, 0\}, H|\{\alpha, 0\})$, in accordance with the Note preceding this theorem. The condition (iii) merely ensures that the entire semigroup $S$ is not royal.

As regards the direct half of the theorem, the only issue is whether the multiplication on $S$ is associative. Let $a \in E_{\alpha}, b \in E_{\beta}, c \in E_{\gamma}$. If $\alpha=\beta=\gamma$ then $(a b) c=a(b c)$ by associativity within a right or left zero semigroup. If $|\{\alpha, \beta, \gamma\}|=2$ then $(a b) c=a(b c)$ by associativity within a royal or singly noble semigroup with two $\mathscr{J}$-classes. If $\alpha, \beta, \gamma$ are all distinct then all cases are automatic by the properties of royal semigroups except

$$
\begin{aligned}
& \alpha>\beta>0, \beta>\alpha>0, \alpha>0>\beta, \beta>0>\alpha, \\
& \alpha>\gamma>0, \gamma>\alpha>0, \alpha>0>\gamma, \gamma>0>\alpha, \\
& \beta>\gamma>0, \gamma>\beta>0, \beta>0>\gamma, \gamma>0>\beta \text {. }
\end{aligned}
$$

This is a tedious verification and it will suffice to give an example. Suppose that $\alpha>\beta>0$ and that $H(\alpha)=H(\beta)=R, H(0)=L$. Then $E_{\alpha}, E_{\beta}$ are right zero semigroups, while $E_{0}$ is left zero. If $a \in E_{\alpha}, b \in E_{\beta}, z \in E_{0}$ then $P(\alpha) \subseteq P(\beta)$ and we have

$$
(a b) z=b z=\left\{\begin{array}{lll}
z_{1} & \text { if } & z \in P(\beta) \\
z & \text { if } & z \notin P(\beta)
\end{array}\right.
$$


On the other hand

$$
\begin{aligned}
a(b z) & =\left\{\begin{array}{lll}
a z_{1} & \text { if } & z \in P(\beta) \\
a z & \text { if } & z \notin P(\beta)
\end{array}\right. \\
& =\left\{\begin{array}{lll}
z_{1} & \text { if } & z \in P(\beta) \\
z & \text { if } & z \notin P(\beta),
\end{array}\right.
\end{aligned}
$$

since $z \notin P(\beta)$ certainly implies $z \notin P(\alpha)$. Thus $(a b) z=a(b z)$ as required. Other cases are either similar or easier.

The singly noble band described in the theorem will be denoted by

$$
S=S N B(Y ; M, H ; P) \text {. }
$$

Remark. If we drop condition (iii) in the statement of the theorem we may have $P(\alpha)=\left\{z_{1}\right\}$ for all $\alpha>0$. In this case $S$ reduces to $\operatorname{Roy}(Y, M, H)$.

In order to study singly noble bands that are not catenary we require some more terminology. A singly noble band will be called feminine if the pivotal $\mathscr{J}$-class consists of a single element, and masculine otherwise. (The feminine bands have narrower waists.) Theorem 4.11 indicates that all catenary singly noble bands are masculine, but we shall see that this need not be so for the non-catenary case.

Let $S=\mathscr{P}\left[Y ;\left\{E_{\alpha}: \alpha \in Y\right\}\right]$ be a singly noble band in which $Y$ is not a chain. Denote the unique branch-point of $Y$ by 0 and the pivot by $z_{1}\left(\in E_{0}\right)$. We shall in fact limit ourselves to the case where $S$ is arboreal, i.e. where the underlying semilattice $Y$ is a tree. The more general case would not be impossible to describe, but the details might be tedious and in order to keep this paper to a reasonable length we shall not tackle it here.

In effect the restriction to the arboreal case means that $\{\alpha \in Y: \alpha \geqq 0\}$ is the union of chains $Z_{i}(i \in I)$, with

$$
Z_{i} \cap Z_{j}=\{0\} \quad(i \neq j)
$$

Since $Y$ is not a chain we must have $|I| \geqq 2$.

Suppose now that $S$ is both masculine and arboreal. We define $M: Y \rightarrow \Omega$ by $M(\alpha)=\left|E_{\alpha}\right|$ and note that $M(0) \geqq 2$. Also, we define $H: Y \rightarrow\{R, L\}$ by the rule that

$$
H(\alpha)=\left\{\begin{array}{lll}
R & \text { if } & E_{\alpha} \in \mathbf{R Z} \\
L & \text { if } & E_{\alpha} \in \mathbf{L Z} \text { and }\left|E_{\alpha}\right|>1 .
\end{array}\right.
$$

For each $i$ in $I$ the structure of the catenary singly noble (or royal) band $S_{i}=\bigcup\left\{E_{\alpha}: \alpha \in Z_{i}\right\}$ determines a map $P_{i}: Z_{i} \rightarrow \mathscr{P}\left(E_{0}\right)$ satisfying conditions (i) and (ii) of Theorem 4.11: that is, $z_{1} \in P_{i}(\alpha)$ for all $\alpha$ in $Z_{i}$; and for all $\alpha, \beta$ in $Z_{i}$

$$
0<\alpha \leqq \beta \Rightarrow P_{i}(\alpha) \supseteq P_{i}(\beta) .
$$


Then

$$
S_{i} \simeq S N B\left(Z_{i} ; M\left|Z_{i}, H\right| Z_{i} ; P_{i}\right)
$$

the case where $S_{i}$ is royal being covered by the eventuality that $P_{i}(\alpha)=\left\{z_{1}\right\}$ for all $\alpha$ in $Z_{i}$.

In fact the functions $P_{i}(i \in I)$ are not independent. To see this, let us look at $\alpha$ in $Z_{i}$, $\beta$ in $Z_{j}$ (with $i \neq j$ ) and let us suppose for definiteness that

$$
H(\alpha)=R, \quad H(\beta)=H(0)=L
$$

Then $E_{\alpha} \cup E_{0}, E_{\beta} \cup E_{0}$ are respectively

$$
S N B_{L}^{R}\left(E_{\alpha}, E_{0} ; P_{i}(\alpha) ; z_{1}\right), \quad S N B_{L}^{L}\left(E_{\beta}, E_{0} ; P_{j}(\beta) ; z_{1}\right)
$$

For $a \in E_{\alpha}, b \in E_{\beta}$ we must have

$$
a b=b a=z_{1} .
$$

since $E_{\alpha} E_{\beta}, E_{\beta} E_{\alpha} \subseteq E_{0}$ and since $z_{1}$ is the unique superfluous element of $S$. Hence for all $z$ in $E_{0}$

$$
(a b) z=z_{1} z=z_{1}
$$

while

$$
\begin{aligned}
& a(b z)=\left\{\begin{array}{lll}
a z_{1} & \text { if } & z \in P_{j}(\beta) \\
a z & \text { if } & z \notin P_{j}(\beta)
\end{array}\right. \\
& = \begin{cases}z_{1} & \text { if } \quad z \in P_{i}(\alpha) \cup P_{j}(\beta) \\
z & \text { otherwise }\end{cases}
\end{aligned}
$$

Thus associativity fails unless

$$
P_{i}(\alpha) \cup P_{j}(\beta)=E_{0}
$$

for all $i \neq j$ and all $\alpha$ in $Z_{i}, \beta$ in $Z_{j}$.

We have therefore proved the converse half of the following theorem:

Theorem 4.14. Let $Y$ be a tree with a single branch-point 0 and suppose that $\{\alpha \in Y: \alpha \geqq 0\}$ is a union of chains $Z_{i}(i \in I)$, with

$$
|I| \geqq 2, \quad\left|Z_{i}\right| \geqq 2(i \in I), \quad Z_{i} \cap Z_{j}=\{0\} \quad(i \neq j) .
$$

Let

$$
S_{i}=S N B\left(Z_{i} ; M_{i}, H_{i} ; P_{i}\right) \quad(i \in I)
$$


be a catenary singly noble or royal band with $\mathscr{J}$-classes $E_{\alpha}\left(\alpha \in Z_{i}\right)$, pivotal $\mathscr{J}$-class $E_{0}$ and pivot $z_{1}$, and suppose that

$$
S_{i} \cap S_{j}=E_{0} \quad(i \neq j) .
$$

Suppose also that for all $i \neq j$ and all $\alpha$ in $Z_{i}, \beta$ in $Z_{j}$,

$$
P_{i}(\alpha) \cup P_{j}(\beta)=E_{0}
$$

Let $\bar{Y}=\{\alpha \in Y: \alpha<0\}$ and let

$$
T=\operatorname{Roy}(\bar{Y}, M, H)
$$

be a royal band, where $T \cap S_{i}=\emptyset$ for all $i$ in $I$.

Define a multiplication on

$$
S=T \cup \bigcup\left\{S_{i}: i \in I\right\}
$$

by the rule that

$$
\begin{gathered}
\left(S_{i} \backslash E_{0}\right)\left(S_{j} \backslash E_{0}\right)=\left\{z_{1}\right\} \quad(i \neq j), \\
x y=y x=y \quad \text { whenever } \quad x \in S_{i}, y \in T .
\end{gathered}
$$

Then $S$ is an arboreal masculine singly noble band.

Conversely, every arboreal masculine singly noble band is isomorphic to one of this kind.

Proof. Here the verification of associativity (which is all that remains to be proved) is much easier than in Theorem 4.11 and is omitted altogether.

For feminine singly noble bands we obtain the following theorem:

Theorem 4.15. Let $R_{i}(i \in I)$ be non-trivial royal bands intersecting in a common minimum $\mathscr{J}$-class $\{z\}$, where $|I| \geqq 2$. Let $R_{0}$ be a royal band whose maximum $\mathscr{J}$-class is $\{z\}$. Let

$$
S=R_{0} \cup \bigcup\left\{R_{i}: i \in I\right\}
$$

and define a multiplication on $S$ by

$$
\begin{gathered}
R_{i} R_{j}=\{z\} \quad(i, j \in I, i \neq j), \\
x y_{0}=y_{0} x=y_{0} \quad\left(x \in R_{i}, i \in I, y_{0} \in R_{0}\right) .
\end{gathered}
$$

Then $S$ is an arboreal feminine singly noble band.

Conversely, every arboreal feminine singly noble band is isomorphic to one constructed in this way. 
Proof. The verification that $S$ is associative is trivial. Also $S$ is singly noble, generated by $S \backslash\{z\}$, and is feminine since the pivotal $\mathscr{J}$-class consists of a single element $z$. It is arboreal, since the underlying semilattice has one branch-point at 0 from which at least two chains diverge upwards.

To establish the converse half, suppose that $S=\mathscr{S}\left[Y ;\left\{E_{\alpha}: \alpha \in Y\right\}\right]$ is an arboreal feminine singly noble band. We know from Theorem 4.11 that a catenary singly noble band must be masculine; hence $Y$ has a branch-point, and this must be at the pivotal $\mathscr{J}$-class $E_{0}=\{z\}$. In our previous notation, write $\{\alpha \in Y: \alpha \geqq 0\}$ as $\bigcup\left\{Z_{i}: i \in I\right\}$, where the $Z_{i}$ are chains and where $Z_{i} \cap Z_{j}=\{0\}$ if $i \neq j$. Then for each $i$ in $I$ the catenary subsemigroup

$$
R_{i}=\bigcup\left\{E_{\alpha}: \alpha \in Z_{i}\right\}
$$

cannot be singly noble (since it is feminine) and so must be royal. This applies also to

$$
R_{0}=\bigcup\left\{E_{\alpha}: \alpha \leqq 0\right\}
$$

The result is now clear.

\section{Singly noble semigroups}

In addition to singly noble bands we have two very obvious sources of singly noble semigroups. First, it is clear that $\mathbf{Z}_{2}$, the two element group, is singly noble, and that it is the only group with this property. Secondly, any null semigroup $N$, with $N^{2}=\{z\}$, is singly noble. It turns out that the structure of singly noble semigroups can be described in terms of singly noble bands, copies of $\mathbf{Z}_{2}$ and null semigroups.

Let $S$ be a singly noble semigroup, generated by $S \backslash\{z\}$. We saw in Section 3 that $z^{2}=z$ and that for each $g$ in $S \backslash\{z\}$ either $g^{2}=g$ or $g^{2}=z$. In fact we have

Lemma 5.1. Let $S$ be a singly noble semigroup, generated by $S \backslash\{z\}$, and let $B=$ $\left\{s \in S: s^{2}=s\right\}$. Then $B$ is a subsemigroup of $S$ and is either a royal band or a singly noble band.

Proof. Let $b, c \in B$. Then

$$
b c \in\{b, c, z\} \subseteq B
$$

and so $B$ is a subsemigroup. The result is now clear.

Lemma 5.2. Let $S$ be a singly noble semigroup, generated by $S \backslash\{z\}$, and suppose that $|C| \geqq 2$, where $C=\left\{s \in S: s^{2}=z\right\}$. Then $C$ is a singly noble subsemigroup of $S$.

Proof. As above, if $s, t \in C$ then

$$
s t \in\{s, t, z\} \subseteq C
$$


and so $C$ is a subsemigroup. Being contained in a singly noble semigroup, it must be either royal or singly noble, but since it is not a band it cannot in fact be royal.

Let us now look a little more closely at $C$. For each $c$ in $C$ we have $c^{2}=z$ and so

$$
c z=z c\left(=c^{3}\right)
$$

Hence $C=G \cup N$, where

$$
\begin{aligned}
& G=\{c \in C: c z=z c=c\}, \\
& N=\{c \in C: c z=z c=z\},
\end{aligned}
$$

and $G \cap N=\{z\}$. Then $G$ is closed under multiplication, since $c, d \in G$ implies

$$
(c d) z=c(d z)=c d, z(c d)=(z c) d=c d,
$$

and in fact $G$ is a group with identity $z$, with $c=c^{-1}$ for all $c$ in $G$. It must be singly noble or royal, being a subsemigroup of a singly noble semigroup, and hence $|G| \leqq 2$. That is, either $G=\{z\}$, or $G=\{z, c\}$, with $c z=z c=c, c^{2}=z^{2}=z$.

Next, we examine $N$. If $c, d \in N \backslash\{z\}$ then $c d \in\{c, d, z\}$. If $c d=c$ then

$$
c=c d=(c d) d=c d^{3}=c z=z,
$$

a contradiction, and we similarly get a contradiction from the assumption that $c d=d$. Hence $c d=z$ and so $N$ is a null semigroup with $z$ as zero element.

We have thus proved

Theorem 5.3. Let $S$ be a singly noble semigroup, generated by $S \backslash\{z\}$. Then there exist a royal or singly noble band $B$, a group $G$ of order not greater than 2 with identity $z$, and a null semigroup $N$ with zero element $z$ such that

$$
B \cap G=B \cap N=G \cap N=\{z\}
$$

and $S=B \cup G \cup N$. If $B$ is royal then at least one of $G, N$ is non-trivial.

Probing more deeply into the fine structure of $S$, let us now suppose that $B$ is masculine, i.e. that the $\mathscr{J}$-class of $z$ in $B$ contains at least one other element $z_{2}$. Let us suppose (without essential loss of generality) that the $\mathscr{J}$-class $\left\{z, z_{2}, \ldots\right\}$ is in $\mathbf{R Z}$, so that

$$
z z_{2}=z_{2}, \quad z_{2} z=z .
$$

Suppose now that $G=\{g, z\}$, of order 2 . Then

$$
z_{2} g=z_{2}(z g)=\left(z_{2} z\right) g=z g=g,
$$


and $g z_{2} \in\left\{g, z_{2}, z\right\}$. We show that each of the three possibilities leads to a contradiction. First, $g z_{2}=g$ gives

$$
z=g^{2}=g^{2} z_{2}=z z_{2}=z_{2}
$$

which is not the case. Secondly, $g z_{2}=z_{2}$ gives

$$
g=g z=g\left(z_{2} z\right)=\left(g z_{2}\right) z=z_{2} z=z
$$

and thirdly, $g z_{2}=z$ gives

$$
g=z g=\left(g z_{2}\right) g=g\left(z_{2} g\right)=g^{2}=z
$$

Our conclusion is as follows.

Theorem 5.4. If in Theorem 5.3 the band $B$ is masculine then the group $G$ is trivial.

It may help in explaining our remaining results if we introduce some additional terminology. We shall say that a singly noble semigroup $S$, generated by $S \backslash\{z\}$, is dexter if the pivotal $\mathscr{I}$-class $J_{z}$ is a right zero semigroup containing at least two elements, sinister if $J_{z}$ is a left zero semigroup containing at least two elements, feminine if $J_{z}=\{z\}$, and balanced if $J_{z}=\{z, g\}$, a group of order 2. It follows from our investigations so far that these are the only possibilities. Notice that the masculine singly noble bands investigated in Theorems 4.11 and 4.14 have either the dexter or the sinister property.

Before stating the next theorem we require a further definition. Let $Y$ be a semilatice with a single branch-point 0 and let $Y^{+}=\{\alpha \in Y: \alpha>0\}$. A subset $C$ of $Y^{+}$will be called a positive chain filter if

(i) $[\alpha \in C, \beta>\alpha] \Rightarrow \beta \in C$,

(ii) $[\alpha \in C, \beta$ not comparable with $\alpha] \Rightarrow \beta \notin C$. $\}$

Such subsets exist: the empty set is one such, and so is every set $[\alpha)=,\{\beta \in Y: \beta \geqq \alpha\}$. Let $\mathscr{C}$ denote the set of all positive chain filters in $Y$.

Theorem 5.6. Let $B=\mathscr{S}\left[Y ;\left\{E_{\alpha}: \alpha \in Y\right\}\right]$ be a dexter singly noble band, generated by $B \backslash\{z\}$ and with pivotal $\mathscr{J}$-class $E_{0}$. Let $N$ be a null semigroup with zero element $z$ but otherwise disjoint from $B$. Let

$$
\Phi_{r}: N \backslash\{z\} \rightarrow \mathscr{C}, \quad \Phi_{l}: N \backslash\{z\} \rightarrow \mathscr{C}
$$

be mappings from $N \backslash\{z\}$ into the set $\mathscr{C}$ of positive chain filters of $Y$. Define a multiplication on $S=B \cup N$ by the rules that

$$
\begin{aligned}
& N b_{\alpha}=b_{\alpha} N=\left\{b_{\alpha}\right\} \quad \text { if } \quad b_{\alpha} \in E_{\alpha}, \quad \alpha<0, \\
& x b_{0}=b_{0}, b_{0} x=z \quad \text { if } \quad x \in N, \quad b_{0} \in E_{0},
\end{aligned}
$$


while if $x \in N \backslash\{z\}, b_{\alpha} \in E_{\alpha}, \alpha>0$,

$$
\begin{aligned}
& b_{\alpha} x=\left\{\begin{array}{lll}
x & \text { if } & \alpha \in \Phi_{r}(x) \\
z & \text { if } & \alpha \notin \Phi_{r}(x),
\end{array}\right. \\
& x b_{\alpha}=\left\{\begin{array}{lll}
x & \text { if } & \alpha \in \Phi_{l}(x) \\
z & \text { if } & \alpha \notin \Phi_{r}(x) .
\end{array}\right.
\end{aligned}
$$

Then $S$ is a dexter singly noble semigroup.

Conversely, every dexter singly noble semigroup is isomorphic to one constructed in this way.

Proof. For the direct part of the theorem the only issue is whether $S$ is a semigroup. Once that is established, the additional properties are clear. The verification of associativity falls into six main cases, labelled (in an obvious notation)

$$
B N N, N B N, N N B, B B N, B N B, N B B \text {. }
$$

For the first three cases it is helpful to look separately at

$$
B^{+}=\bigcup\left\{E_{\alpha}: \alpha>0\right\}, B^{-}=\bigcup\left\{E_{\alpha}: \alpha<0\right\} \text { and } E_{0}
$$

Since $N^{2}=\{z\}$ and $B^{+} N, N B^{+} \subseteq N$ we see that in cases $B^{+} N N, N B^{+} N$ and $N N B^{+}$both products $(p q) r$ and $p(q r)$ must equal $z$. In case $B^{-} N N$ the rules give

$$
(p q) r=p(q r)=p
$$

and cases $N B^{-} N, N N B^{-}$are equally straightforward. In cases $E_{0} N N$ and $N E_{0} N$ it is easy to see that

$$
(p q) r=p(q r)=z
$$

while in case $N N E_{0}$ we have

$$
(p q) r=p(q r)=r
$$

For the case $B B N$, and indeed in a similar way for each of the remaining cases, it is necessary to consider the products $(b c) x$ and $b(c x)$ (with $b \in E_{\beta}, c \in E_{\gamma}, x \in N$ ) case by case as follows:
(i) $\beta>\gamma>0$
(ii) $\beta=y>0$,
(iii) $\gamma>\beta>0$,
(iv) $\beta>0>\gamma$,
(v) $\gamma>0>\beta$,
(vi) $0>\beta>\gamma$,
(vii) $0>\beta=\gamma$,
(viii) $0>\gamma>\beta$,
(ix) $\beta>\gamma=0$,
(x) $\gamma>\beta=0$,
(xi) $0=\beta>\gamma$,
(xii) $0=\gamma>\beta$,
(xiii) $\beta=\gamma=0$. 
This is a tedious procedure and we shall record only a couple of cases where there is special interest. First, in case (i) we have

$$
(b c) x=c x= \begin{cases}x & \text { if } \gamma \in \Phi_{r}(x) \\ z & \text { otherwise }\end{cases}
$$

while

$$
\begin{aligned}
b(c x) & = \begin{cases}b x & \text { if } \quad \gamma \in \Phi_{r}(x) \\
b z & \text { otherwise }\end{cases} \\
& = \begin{cases}x & \text { if } \gamma \in \Phi_{r}(x) \\
z & \text { otherwise }\end{cases}
\end{aligned}
$$

since $\beta>\gamma$ and $\gamma \in \Phi_{r}(x)$ gives $\beta \in \Phi_{r}(x)$ by (5.5). Thus $(b c) x=b(c x)$.

In case $(x)$ we note that $b c \in\{b, z\}$. Hence $(b c) x \in\{b x, z x\}$ and so by $(5.7)(b c) x=z$. For the other product, note that $c x=x$ if $y \in \Phi_{r}(x)$ and is otherwise equal to $z$. Hence $b(c x) \in\{b x, b z\}$ and so, by (5.7) and the right zero property of $E_{0}$,

$$
b(c x)=z .
$$

None of the 39 cases is harder than these, and most are easier.

Turning now to the converse part, we know from Theorems 5.3 and 5.4 that if $S$ is a dexter singly noble semigroup generated by $S \backslash\{z\}$ then $S=B \cup N$, where $B$ is a dexter singly noble band with $z$ as pivot, $N$ is a null semigroup with zero element $z$, and $B \cap N=\{z\}$. The structure of $B=\mathscr{S}\left[Y ;\left\{E_{\alpha}: \alpha \in Y\right\}\right]$ is given by Theorem 4.14. Denote the pivotal $\mathscr{J}$-class of $B$ by $E_{0}=\left\{z, z_{2}, \ldots\right\}$. We now show that the multiplication rules in $B \cup N$ must be as listed in the statement of the theorem.

First, let $x \in N \backslash\{z\}$ and suppose that $b_{\alpha} \in E_{\alpha}, \alpha<0$. Then $x b_{\alpha} \in\left\{x, b_{\alpha}, z\right\}$. If $x b_{\alpha}=x$ then

$$
z=x^{2}=x^{2} b_{\alpha}=z b_{\alpha}
$$

in contradiction to the multiplication rules in $B$; if $x b_{\alpha}=z$ then again we have a contradiction, since

$$
z=x b_{\alpha}=x b_{\alpha}^{2}=\left(x b_{\alpha}\right) b_{\alpha}=z b_{\alpha}
$$

hence $x b_{\alpha}=b_{\alpha}$. Thus $N b_{\alpha}=\left\{b_{\alpha}\right\}$, and similarly $b_{\alpha} N=\left\{b_{\alpha}\right\}$.

Next, let $x \in N \backslash\{z\}$ and suppose that $b_{0} \in E_{0} \backslash\{z\}$. Then $x b_{0} \in\left\{x, b_{0}, z\right\}$. If $x b_{0}=x$ then the right zero property of $E_{0}$ gives

$$
z=x^{2}=x^{2} b_{0}=z b_{0}=b_{0},
$$

a contradiction; if $x b_{0}=z$ then we again have a contradiction, since

$$
z=x b_{0}=x b_{0}^{2}=\left(x b_{0}\right) b_{0}=z b_{0}=b_{0}
$$


hence we must have

$$
x b_{0}=b_{0} .
$$

Turning now to $b_{0} x$, which also belongs to $\left\{x, b_{0}, z\right\}$, we see that $b_{0} x=x$ gives

$$
x=b_{0} x=\left(x b_{0}\right) x=x\left(b_{0} x\right)=x^{2}=z
$$

a contradiction. If we assume $b_{0} x=b_{0}$ we obtain

$$
b_{0}=b_{0} x=b_{0} x^{2}=b_{0} z=z,
$$

again a contradiction. Hence $b_{0} x=z$.

Suppose now that $x \in N \backslash\{z\}$ and that $b_{\alpha} \in E_{\alpha}, \alpha>0$. Then $b_{\alpha} x=\left\{b_{\alpha}, x, z\right\}$. If $b_{\alpha} x=b_{\alpha}$ then

$$
b_{\alpha}=b_{\alpha} x=b_{\alpha} x^{2}=b_{\alpha} z
$$

a contradiction to the multiplication properties of $B$; hence

$$
b_{\alpha} x=x \quad \text { or } \quad b_{\alpha} x=z
$$

For a given $x$ in $N \backslash\{z\}$ there are now just two possibilities: either $b x=z$ for all $b$ in $\bigcup\left\{E_{\alpha}, \alpha>0\right\}$, in which case we define

$$
\Phi_{r}(x)=\emptyset
$$

or there exists $\delta>0$ and $b_{\delta} \in E_{\delta}$ for which $b_{\delta} x=x$. Suppose that the latter event occurs, and let $c_{\delta} \in E_{\delta}$. Then $c_{\delta} x \in\{x, z\}$. If $c_{\delta} x=z$ we obtain a contradiction: if $E_{\delta} \in \mathbf{R Z}$ we have

$$
z=c_{\delta} x=c_{\delta}\left(b_{\delta} x\right)=\left(c_{\delta} b_{\delta}\right) x=b_{\delta} x=x
$$

while if $E_{\delta} \in \mathbf{L Z}$ we have

$$
x=b_{\delta} x=\left(b_{\delta} c_{\delta}\right) x=b_{\delta}\left(c_{\delta} x\right)=b_{\delta} z=z
$$

Hence $c_{\delta} x=x$.

If as before we write

$$
B^{+}=\bigcup\left\{E_{\alpha}: \alpha>0\right\}
$$

We can deduce from the result just proved that the set $\left\{b \in B^{+}: b x=x\right\}$ is a union

$$
\bigcup\left\{E_{a}: \alpha \in C\right\}
$$

of $\mathscr{J}$-classes, where $C \subseteq Y^{+}$. We now show that $C$ is a positive chain filter. 
First, let $\alpha \in C$ and let $\beta>\alpha$. Then for all $c_{\beta}$ in $E_{\beta}$

$$
\begin{aligned}
c_{\beta} x & =c_{\beta}\left(b_{\alpha} x\right) \quad\left(\text { where } b_{\alpha} \in E_{\alpha}\right) \\
& =\left(c_{\beta} b_{\alpha}\right) x=b_{\alpha} x=x .
\end{aligned}
$$

Hence $\beta \in C$.

Next, let $\alpha \in C$ and suppose that $\beta$ is not comparable with $\alpha$. Then $E_{\alpha} E_{\beta}=\{z\}$ and we must have $c_{\beta} x=z$ for all $c_{\beta}$ in $E$, since $c_{\beta} x=x$ (the only other possibility) implies

$$
\begin{aligned}
x & =b_{\alpha} x \quad\left(\text { where } b_{\alpha} \in E_{\alpha}\right) \\
& =b_{\alpha}\left(c_{\beta} x\right)=\left(b_{\alpha} c_{\beta}\right) x=z x=z,
\end{aligned}
$$

a contradiction. Hence $\beta \notin C$.

The conclusion is that for a given $x$ in $N \backslash\{z\}$ either $b x=z$ for all $b$ in $B^{+}$, giving

$$
\Phi_{r}(x)=\emptyset
$$

as already remarked, or there exists a unique non-empty positive chain filter $C$ such that

$$
\left\{b \in B^{+}: b x=x\right\}=\bigcup\left\{E_{x}: \alpha \in C\right\} .
$$

In this case we define $\Phi_{r}(x)=C$.

In exactly the same way we can consider $x b$ and obtain a mapping $\Phi_{l}: N \backslash\{z\} \rightarrow \mathscr{C}$ such that

$$
\left\{b \in B^{+}: x b=x\right\}=\bigcup\left\{E_{\alpha}: \alpha \in \Phi_{l}(x)\right\}
$$

This completes the proof.

We also have a dual theorem, in which dexter is replaced by sinister throughout and (5.7) becomes

$$
x b_{0}=z, b_{0} x=b_{0} \quad \text { if } \quad x \in N, b_{0} \in E_{0}
$$

The case of a feminine singly noble semigroup is also effectively dealt with: in Theorem 5.5 simply replace dexter by feminine throughout and replace (5.7) by

$$
x z=z x=z \quad \text { if } \quad x \in N .
$$

(In this case $E_{0}=\{z\}$.)

The remaining case, that of a balanced singly noble semigroup, is now disposed of fairly easily. 
Theorem 5.10. Let $F=B \cup N$, with $B=\mathscr{S}\left[Y ;\left\{E_{a}: \alpha \in Y\right\}\right]$, be a feminine singly noble semigroup, with pivotal $\mathscr{J}$-class $E_{0}=\{z\}$, and let $g$ be an element not in $F$. Define a multiplication on $S=F \cup\{g\}$ by the rules that

$$
\begin{gathered}
g^{2}=z, g N=N g=\{g\}, \\
g E_{\alpha}=E_{\alpha} g=g \quad \text { if } \quad \alpha>0, \\
g b=b g=b \quad \text { if } \quad b \in E_{\alpha}, \alpha<0 .
\end{gathered}
$$

Then $S$ is a balanced singly noble semigroup. Conversely, every balanced singly noble semigroup is isomorphic to one of this kind.

Proof. Again the direct part of the proof is a routine associativity verification, but this time the verification is much easier and we omit the details.

As regards the converse part, suppose that $S$ is a balanced singly noble semigroup with $\{g, z\}$ as pivotal $\mathscr{J}$-class. By Theorem 5.3 we have $S=B \cup N \cup\{g\}$, where $F=B \cup N$ is a feminine singly noble semigroup with $\{z\}$ as pivotal $\mathscr{J}$-class. We now show that the multiplication rules on $F \cup\{g\}$ must be as listed in the theorem. We know of course that

$$
g z=z g=g, g^{2}=z .
$$

Now let $x \in N \backslash\{z\}$. Then $g x \in\{g, x, z\}$. If $g x=x$ then

$$
z=x^{2}=g x^{2}=g z=g
$$

a contradiction; if $g x=z$ then

$$
z=z x=g^{2} x=g(g x)=g z=g,
$$

again a contradiction; hence

$$
g x=g,
$$

and similarly $x g=g$. Thus

$$
g N=N g=g .
$$

Next, let $b \in E_{\alpha}, \alpha>0$. Then since $J_{g b} \leqq J_{g}=J_{z}<J_{b}$ we must have that

$$
g b \in\{g, z\} .
$$

However, $g b=z$ implies

$$
z=z^{2}=(g b) z=g(b z)=g z=g,
$$


a contradiction, and so in fact $g b=g$. A similar argument establishes that $b g=g$. Thus

$$
g E_{\alpha}=E_{\alpha} g=\{g\}
$$

Finally, let $b \in E_{\alpha}, \alpha<0$. Then since $g b \in\{g, b, z\}$ and

$$
J_{g b} \leqq J_{b}<J_{z}=J_{g}
$$

we must in fact have $g b=b$. By the same argument $b g=b$. This completes the proof.

\section{REFERENCES}

1. A. H. Clifford, Semigroups admitting relative inverses, Ann. of Math. 42 (1941), 1037-1049.

2. J. M. HowIE, An introduction to semigroup theory (Academic Press, London, 1976).

3. J. M. HowIE, Embedding semigroups in semibands: some arithmetical results, Quart. J. Math. Oxford (2) 32 (1981), 323-337.

4. D. McLean, Idempotent semigroups, American Math. Monthly 61 (1954), 110-113.

5. M. Petrich, Lectures in semigroups (Akademie-Verlag, Berlin, 1977).

Departamento de Matemática

Faculdade de Ciencias

UNIVERSIDADE DE LISBOA

1700 Lisboa, Portugal
Mathematical Institute UNIVERSITY OF ST ANDREWS NorTh HAUGH St Andrews, Scotland 